\title{
Properties of phase-shifting devices used in researches of nonlinear absorption
}

\author{
M.R. Kulish, N.I. Malysh \\ V. Lashkaryov Institute of Semiconductor Physics, NAS of Ukraine, \\ 45, prospect Nauky, 03028 Kyiv, Ukraine \\ E-mail:n_kulish@yahoo.com
}

\begin{abstract}
Formulated in this work are the requirements to the Fresnel rhomb used for light polarization control of high-power lasers. Technical parameters of the rhomb have been given. The calibration procedure of the Fresnel rhomb has been described. The single rhomb is proposed to be utillized for the change of light ellipticity from zero to unity, and block of two rhombi for the turn of polarization plane between zero to 360 degrees.
\end{abstract}

Keywords: Fresnel rhomb, wave plate, spectral region, polarization, retarder, ellipticity.

Manuscript received 26.02.09; accepted for publication 18.03.09; published online 20.03.09.

\section{Introduction}

Operation of optical processors [1], switches [2], logical elements [3], autocorrelators [4,5] and other bistabile elements $[5,6]$ based on the using of two-photon absorption. Uniaxial crystals can be utilized as material for these devices [6]. If azimuth of polarization $\varphi \neq m \pi / 2$ ( $\varphi$ is the angle between the crystal optical axis $C$ and the vector $\mathbf{E}$ of electromagnetic wave, $m=$ $0,1,2,3, \ldots)$, in a crystal two light beams spread in the same direction (ordinary with $E \perp C$ and extraordinary with $E \| C$ waves). At presence of nonlinear absorption, the value of intensity in these beams and value of difference between phase of ordinary and extraordinary waves depend on the nonlinear absorption coefficient. So, nonlinear absorption influences on the rotation of the polarization plane and/or value of light ellipticity. It means that the parameters of above devices depend on the intensity and state of light polarization. To predict these changes, it is needed to research the features of influence of the light polarization state on the value of nonlinear absorption. It can be carried out by phase (wave) plates and Fresnel rhomb. To choose a specific type of device capable to control light polarization, it is necessary to know parameters and requirements that should be met by this device. These requirements are formulated below, and it was established that they are best of all satisfied using the Fresnel rhomb. A theory of rhomb operation is shortly considered. There also considered are technical characteristics of rombi and calibration procedure.

\section{Comparison of properties of phase (wave) plates and Fresnel rhomb}

Polarization devices must provide: 1 ) rotation of the polarization plane by $360^{\circ} ; 2$ ) change ellipticity from 0 to $1 ; 3)$ monitoring of the light polarization in a wide spectral range; 4) minimum size of nutation; 5) independence of device properties from the light intensity.

Both the achromatic phase plates and the Fresnel rhomb provide rotation of the polarization plane by $360^{\circ}$ and change of ellipticity from 0 to 1 .

Wave plates and Fresnel rhomb are made with certain tolerance on parallelism and on the angle at rhomb basis. Parallelism tolerance in the zeroorder phase plates $\leq 0.5^{\prime \prime}$ [7], high order $-\leq 1$ " [8], achromatic phase plates $-\leq 10$ " [8]. In rhomb parallelism tolerance $\leq 2$ " [9], angle tolerance at rhomb basis $-\leq 3^{\prime}[10]$. The availability of these tolerances means that wave plates and rhomb can be approximated by a rectangular prism (Fig. 1) with the angle of refraction $\zeta$. For phase plates, the refraction angle equals parallelism tolerance of face, for the Fresnel rhomb - to the sum of tolerance for the angle at rhomb basis and that for parallelism of reflective faces. From this prism, light goes out under an angle $\vartheta$

$$
\vartheta=\operatorname{arc}\left(\frac{\sin \zeta}{n_{21}}\right) \text {. }
$$

The nutation angle is as follows:

$\Delta=\vartheta-\zeta$ 


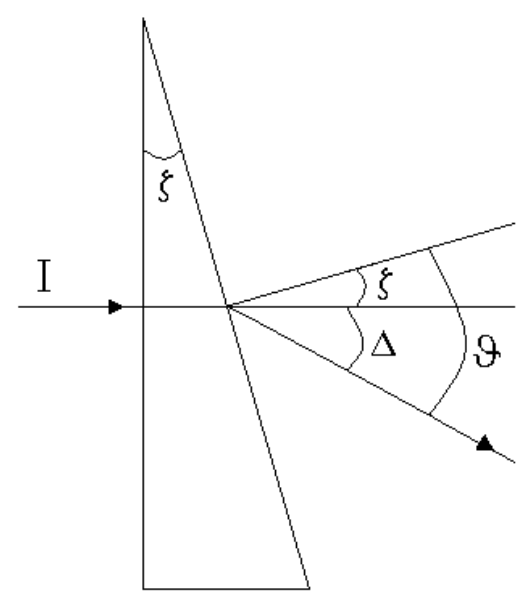

Fig. 1. Illustration to appearance of light beam nutation as a result of light intensity $I$ passing through the Fresnel rhomb. $\zeta$ is the angle of prism refraction which equals to the sum of tolerances on parallelness for operation verges of the rhomb and angle at its basis; $\vartheta$ - angle of prism refraction; $\Delta$ is the angle of nutation. Arrows indicate the way of light beam propagation.

To take account of the above mentioned tolerance information, we estimated a typical value of the nutation angle at the wavelength $\lambda=632.8 \mathrm{~nm}$. In particular, for the zeroorder phase plate $\Delta=0.3^{\prime \prime}$; for the high-order phase plate $\Delta=0.6 "$; for an achromatic phase plate $\Delta=5.5^{\prime \prime}$. For the single rhomb made of BK7 glass $\Delta=1 ' 23 "$; for the double rhomb $\Delta=2^{\prime} 47 "$. It should be noticed that the nutation angle in rhomb mainly depends on the tolerance for the angle at rhomb basis. At large values of tolerance for this angle, the nutation angle can reach the value 30' [9].

Two-photon absorption are researched in a spectral interval $E_{g} / 2<h v<E_{g}$ ( $E_{g}$ is the bandgap value, $h v$ is the energy of light quanta). For example, for $\mathrm{GaN}$ it extends from 340 up to $690 \mathrm{~nm}$, and for GaAs - from 870 up to $1730 \mathrm{~nm}$. Both for the achromatic phase plates and simple Fresnel rhomb, the spectral bandwidth, within the limits of which it is possible to use the unique calibration table, approximately equals $300 \mathrm{~nm}$. Nevertheless, there developed are the rhombi for which in the spectral range of 370 to $1000 \mathrm{~nm}$ a single calibration table is utilized [11].

At light intensities over $1 \mathrm{MW} / \mathrm{cm}^{2}$ (when the laser pulse duration is equal to few tens of nanoseconds), it needs to be taken into account the different influence of two-photon absorption on the intensity of light component in phase plates and Fresnel rhomb as well as on the value of plane-of-polarization rotation. A decrease in the influence of nonlinear absorption in materials of rhomb and phase plates is possible due to operation in the spectral range where $h v<E_{g} / 2$, or due to decrease in the light intensity when dimension of light beam is increased.

This short analysis indicates that the basic properties of phase plates and Fresnel rhomb are similar. However, to research polarization properties for twophoton absorption in a wide spectral interval advantage needs to be given to the Fresnel rhomb, originating from the following reasonings. The phase retardation strongly depends on temperature [12], while the properties of the Fresnel rhomb are not sensitive to temperature changes [13]. The quarter-wave plate is sensitive to deviation of the light beam from the angle of normal incidence [12]. The double Fresnel rhomb (an analogue of quarter-wave plate) is not sensitive to such deviation [14].

\section{Fresnel rhomb}

The properties of the Fresnel rhomb are based on the laws of internal reflection. If the incidence angle $\theta$ (Fig. 2) of the plane-polarized light exceeds the angle of internal reflection, in the reflected beam two waves spread with the mutually perpendicular orientation of $\mathbf{E}$ vector ( $s$ - and $p$-components of light beam) in the same direction. The difference of phases between these waves is possible to be estimated by the formula [15]

$\operatorname{tg} \frac{\delta}{2}=\frac{\cos \theta \sqrt{\sin ^{2} \theta-n_{21}^{2}}}{\sin ^{2} \theta}$,

where $n_{21}$ is the refraction index of more dense medium relatively to the less dense one. The maximal change of phase $\delta_{\max }$ depends only on $n_{21}$

$$
\operatorname{tg} \frac{\delta_{\max }}{2}=\frac{1-n_{21}^{2}}{2 n_{21}} \text {. }
$$

In most of materials, the value of angle phase retardation, which equals to $\pi / 2$, will be realized only after two internal reflections. As a result, the device for light polarization control has the rhombic shape (Fig. 2).

If linearly polarized light falls on the input Fresnel rhomb face, then at $\delta=\pi / 2$ circularly polarized light goes out from it (Fig. 2) and vice versa. Therefore, the rhomb operates as a quarter-wave plate. Using it, we can change the ellipticity from zero (plane-polarized light) to unity (circularly polarized light) (Fig. 2). Two consistently placed rhombi (Fig. 2) operate as half-wave phase plate. Indeed, the first rhomb converts the planepolarized light to elliptically polarized light, and the second - elliptically polarized light to plane-polarized light. Rotating the double rhomb around the light beam axis allows to rotate the polarization plane by $360^{\circ}$.

\subsection{Material for rhomb}

Rhombi are manufactured from optically perfect homogeneous materials of large size with the known dispersion of refraction index. Linear and nonlinear absorption as well as scattering of light in the area of transparency for these materials must be minimum. Therefore, the rhombi are mainly produced from fused silica (operation range equals 190-2500 nm) [16] and glasses of the types BK7 [16] or K8 (operation range equals 360 to $2000 \mathrm{~nm}$ ). For the infra-red region, the rhombi are mainly manufactured from zinc selenide ( $\mathrm{ZnSe})$ for the range 6 to $14 \mu \mathrm{m}$, lead telluride (CdTe) and KRS-5 for the range 6 to $18 \mu \mathrm{m}[14]$. 

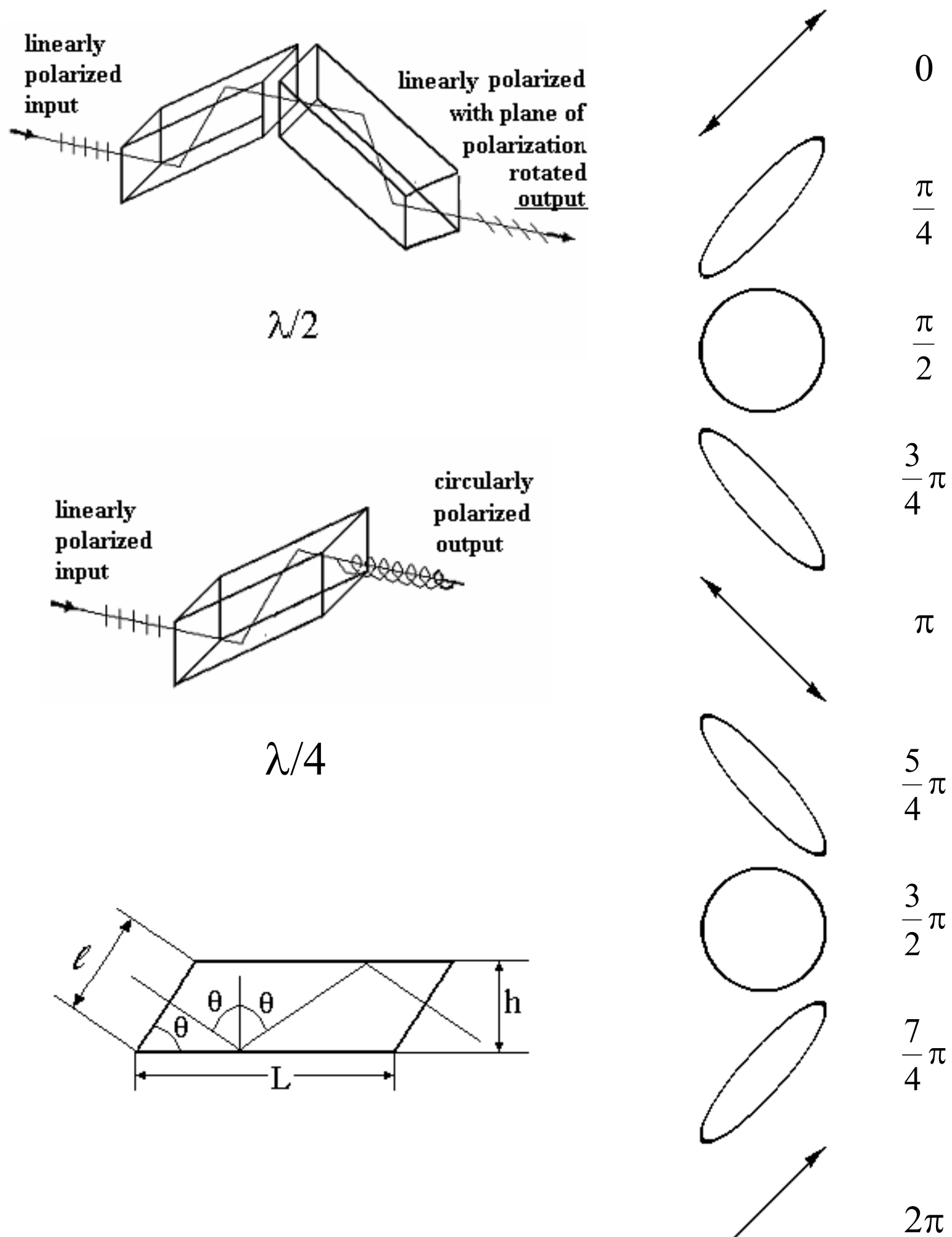

Fig. 2. Illustration to operation of the double (phase retardation $\lambda / 2$ ) and single (phase retardation $\lambda / 4$ ) Fresnel rhombi and of influence of phase retardation (from 0 to $2 \pi$ ) on the shape of polarization ellipse as well as its look. 


\subsection{Technical parameters of Fresnel rhombi}

Let us illustrate the rhomb technical parameters being based on the $\mathrm{K} 8$ glass example. The tabular dependence of the K8 glass refraction index versus wavelength is known [17], which permits to find the dependence $\delta_{\max }$ versus wavelength (Fig. 3) by the formula (4). The wavelength, at exceeding of which $\delta_{\max }$ becomes less $45^{\circ}$, limits the spectral range of rhomb operation like to that the quarter-wave plate does from the side of long waves. The spectral range of double rhomb operation as a half-wave plate from the side of long waves is limited by the presence of vibrational absorption bands. The edge of fundamental absorption band limits the spectral range of rhomb operation from the side of short waves. In the wavelength range, where $\delta_{\max } \geq 45^{\circ}$, we choose the certain wavelength and find $n_{21}$ for it. Substituting $n_{21}$ and $\lambda$ in the formula (3), we estimate the angle of light incidence onto reflecting boundary of the rhomb. In particular, for $\delta=\delta_{\max }=45^{\circ}$ one has $\theta=53^{\circ} 50^{\prime}$ and $\theta=49^{\circ} 46^{\prime}$. Since the angle of incidence must exceed the angle of internal reflection, we choose a greater value of $\theta$. Farther, we substitute $\theta=53^{\circ} 50^{\prime}$ and $n_{21}$ [17] in the formula (3) and find the phase retardation dependence $\delta$ on the wavelength for the double Fresnel rhomb (Fig. 4). The spectral region, where the tolerance for $\delta$ is less than $\pm 0.5^{\circ}$, equals $630 \mathrm{~nm}$. In this spectral interval, it is possible to use the unique calibration table.

Majority of lasers has the Gauss distribution of their intensity in the cross-section of light beam, i.e.

$I=I_{m} \exp \left[-\left(r / r_{0}\right)^{2} \ln 2\right]$,

where $r$ is the distance from the center of light beam, $r_{0}$ is a radius at the level $I=I_{m} / 2, I_{m}$ is the value of intensity at $r=0$. In spectroscopic researches, we usually neglect the light intensity when $I \leq I_{m} / e^{4}$. The value $I=I_{m} / e^{4}$ is realized at the distance from the beam center $r_{e}=2 r_{0} / \sqrt{\ln 2}$. It means that, for the low limit of every

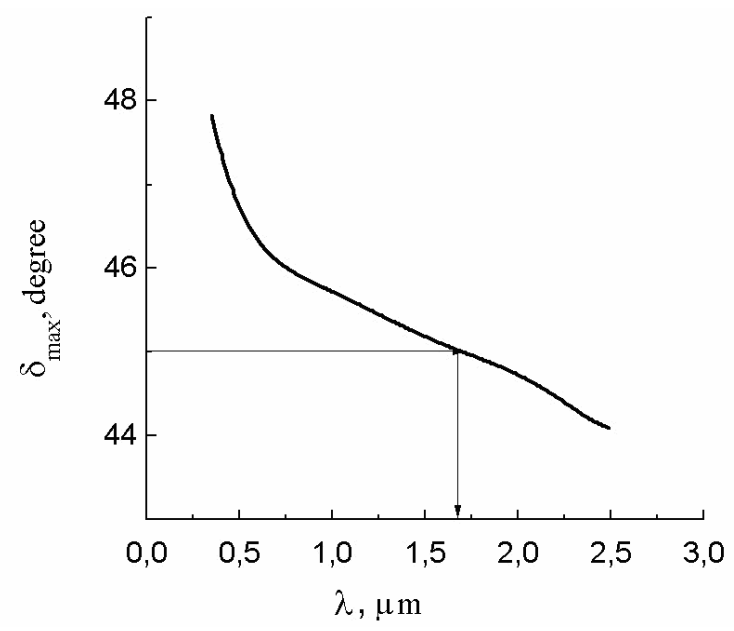

Fig. 3. Wavelength dependence of $\delta_{\max }$. The longwave limit of the region where the single Fresnel rhomb made of $\mathrm{K} 8$ glass can be utillized for the change of light ellipticity, marked by the vertical arrow.

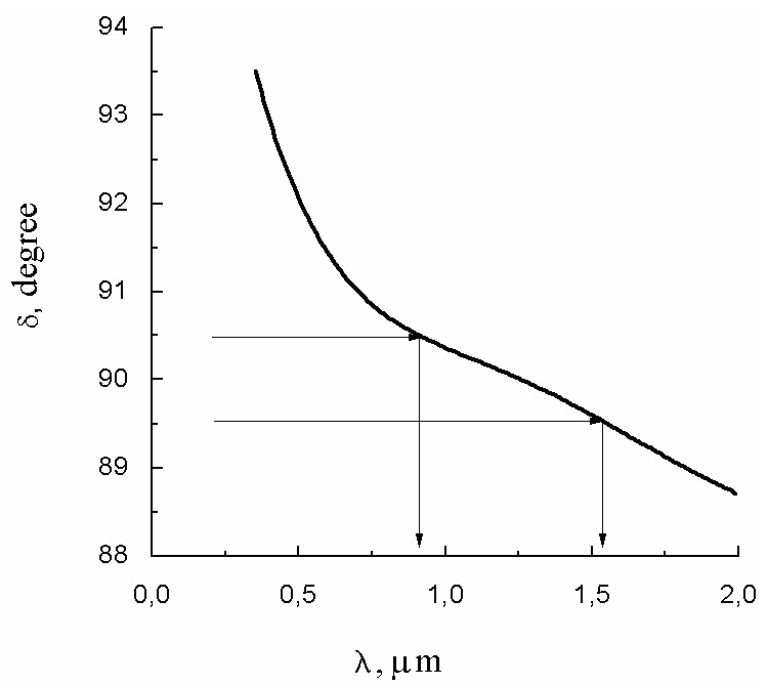

Fig. 4. Phase retardation versus the wavelength for the double Fresnel rhomb. Vertical arrows limit the spectral region where the change of phase retardation angle is less than $\pm 0.5^{\circ}$.

side of square input face of the rhomb, it is possible to take the value $l=2 r_{e}=4 r_{0} / \sqrt{\ln 2}$. According to Fig. 2, the height of this rhomb is $h=l \sin \theta$, and the length of basis is $L=2 h \operatorname{tg} \theta$. These relations allow to calculate overall dimensions of the Fresnel rhomb made of K8 glass (Table 1) and determine their dependence on the beam cross-section of laser light. Other technical parameters of the Fresnel rhomb of K8 glass are summarized in Table 2. In the same table, we show parameters of the Fresnel rhomb made of BK7 glass.

\subsection{Sources of errors in the Fresnel rhombi}

At the choice of a specific construction type for the rhomb, advantage needs to be given to those for which the sum of random and systematic errors when measuring the retardation angle is minimum. The value of random errors can be minimized by an optimum choice of the rhomb construction, his holder and system registering the light intensity. Uncertainties in the angle of light incidence on the entrance face, temperature dependence of the refraction index, dependence of the retardation angle on wavelength; presence of reflection from the entrance and output faces, twist of rhombs are the reasons of linear systematic errors.

Table 1. Overall dimensions of the Fresnel rhomb made of K8 glass.

\begin{tabular}{|c|c|c|c|}
\hline $\begin{array}{c}D_{0}=2 r_{0}, \\
\mathrm{~mm}^{*}\end{array}$ & $l, \mathrm{~mm}$ & $h, \mathrm{~mm}$ & $L, \mathrm{~mm}$ \\
\hline 3 & 7.2 & 5.8 & 15.3 \\
\hline 10 & 24.0 & 19.4 & 51.2 \\
\hline 15 & 36.0 & 29.1 & 76.8 \\
\hline
\end{tabular}

$* D_{\mathrm{o}}$ is the diameter of laser light beam at the level $I=I_{m} / 2$. 
Table 2. Parameters of the glass Fresnel romb.

\begin{tabular}{|l|c|c|}
\hline \multirow{2}{*}{ Material } & \multicolumn{2}{|c|}{ Parameter } \\
\cline { 2 - 3 } & Glass BK7 & Glass K8 \\
\hline $\begin{array}{l}\text { Wavelength range, } \\
\text { nm }\end{array}$ & $\approx 300[16]$ & 630 \\
\hline Angle, degree & $54.6[10]$ & 53.8 \\
\hline $\begin{array}{l}\text { Angle tolerance, arc } \\
\text { min }\end{array}$ & $3[10]$ & 1 \\
\hline $\begin{array}{l}\text { Dimension } \\
\text { tolerance, mm }\end{array}$ & $\pm 0.1[10]$ & \pm 0.1 \\
\hline $\begin{array}{l}\text { Parallelism } \\
\text { tolerance, arc sec }\end{array}$ & $<2[9]$ & $<2$ \\
\hline $\begin{array}{l}\text { Retardation error, } \\
\text { degree }\end{array}$ & $\pm 2[16]$ & \pm 0.5 \\
\hline Antireflection, \% & $\leq 0.2$ at the central & $\leq 0.5$ at $\lambda=$ \\
wavelength [18] & $632.8 \mathrm{~nm}$ \\
\hline Deviation, degree & $\pm 0.5[9]$ & $\pm 0.5 \mathrm{at} \lambda=$ \\
\hline $\begin{array}{l}\text { Max. peak power, } \\
\text { MW/cm }\end{array}$ & $200[19]$ & 200 \\
\hline
\end{tabular}

\subsubsection{Incidence angle}

According to the formula (3), the change of the incidence angle $\theta$ causes a change in the retardation angle. As a result, there is a high sensitivity of the single Fresnel rhomb to deviation of the incidence angle (at the level of angular minutes) from the normal one [14]. It needs to be taken into account when using the single Fresnel rhomb to control the ellipticity of the real light beams, because they always something diverges. The double rhombi are less sensitive to variation of the incidence angle, as the increase of the incidence angle through two complete internal reflections in the first rhomb is compensated by decrease of him due to two complete internal reflections in the second rhomb. As a result, the influence of incidence angle on the phase retardation value is eliminated.

In the double rhomb, there always exists the probability of appearance of some angle between the planes of falling of rhomb, which must influence on the phase retardation angle value. At typical tolerances for the angle at the rhomb basis (angle $\theta$ ) and for rhomb mounting, the angle between output face of the first rhomb and entrance face of the second rhomb varies between $0.2^{\circ}$ and $0.8^{\circ}$. The difference in positions of planes of falling leads to error of the retardation angle less than $0.002^{\circ}$.

\subsubsection{Reflection from entrance and output faces}

We can consider every rhomb as a Fabry-Perot etalon, if its entrance and output faces are rigorously parallel. The tolerances for parallelness of entrance and output faces as well as for the angle at the rhomb basis (Table 2) have the values that allow to neglect interference effects in the rhomb. The presence of antireflecting layers on the entrance and output faces also promotes to neglect these interference effects.

\subsubsection{Influence of temperature}

In the Fresnel rhomb, phase retardation depends on $n_{12}$, and $n_{12}$, in its turn, depends on temperature. In most of optically homogeneous media, the temperature dependence of $n_{12}$ is not great. In particular, at room temperatures for K8 glass, for BK7 glass and for fused silica $n_{12} / d T$ equals $2.8 \cdot 10^{-6} /{ }^{\circ} \mathrm{K}[20], 2.4 \cdot 10^{-6} /{ }^{\circ} \mathrm{K}$, $11.9 \cdot 10^{-6} /{ }^{\circ} \mathrm{K}$ [22], respectively. For ZnSe, CdTe, KRS-5 at $\lambda=10.6 \mu \mathrm{m}, n_{12} / d T$ respectively equals $61 \cdot 10^{-6} /{ }^{\circ} \mathrm{K}$, $107 \cdot 10^{-6} /{ }^{\circ} \mathrm{K},-99 \cdot 10^{-6} /{ }^{\circ} \mathrm{K}[23]$. According to these data for $n_{12} / d T$, the change of rhomb temperature by $\pm 10^{\circ}$ produces the changes of $n_{12}$ does not exceed unity in the third order after the decimal point. It means that the change of rhomb temperature does not practically influence on the phase retardation value.

\section{Rhomb calibration}

The purpose of calibration consists in finding the table and/or analytical dependence of position for polarization plane on the output of the double Fresnel rhomb and change of light ellipticity on the output of single rhomb versus angular position of the polarization plane at their entrance. Typical block diagram for rhomb calibration is shown in Fig. 5.

The testing stages of double Fresnel rhomb calibration are as follows.

1) The light beam from a helium-neon laser is directed to photodetectors that check $I_{0}$ and $I$, accordingly.

2) Polarizer is inserted. As light of the helium-neon laser is polarized, rotating the polarizer we find the position when $I$ is maximal. Fix this angular position.

A
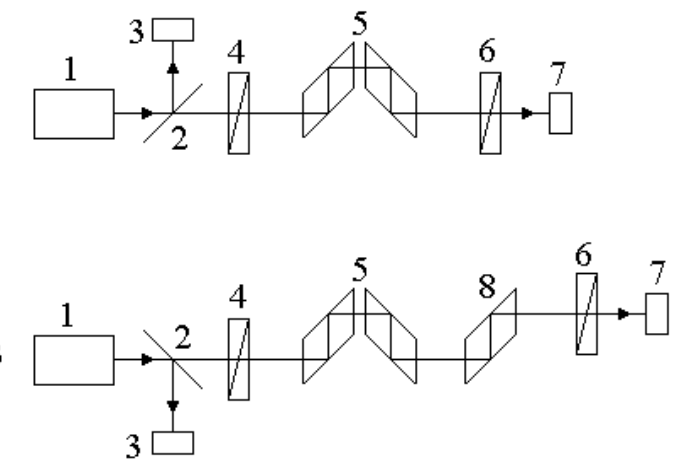

Fig. 5. Experimental setup for calibration of double (A) and single (B) Fresnel rhombi. 1 - He-Ne laser, 2 - coupler, 3 photodetector (photodiode FD-24K) that controls $I_{0}, 4-$ polarizer (Glan prism), 5 - double Fresnel rhomb, 6 - analyzer (Glan prism), 7 - photodetector (photodiode FD-24K) that controls $I, 8$ - single Fresnel rhomb. 

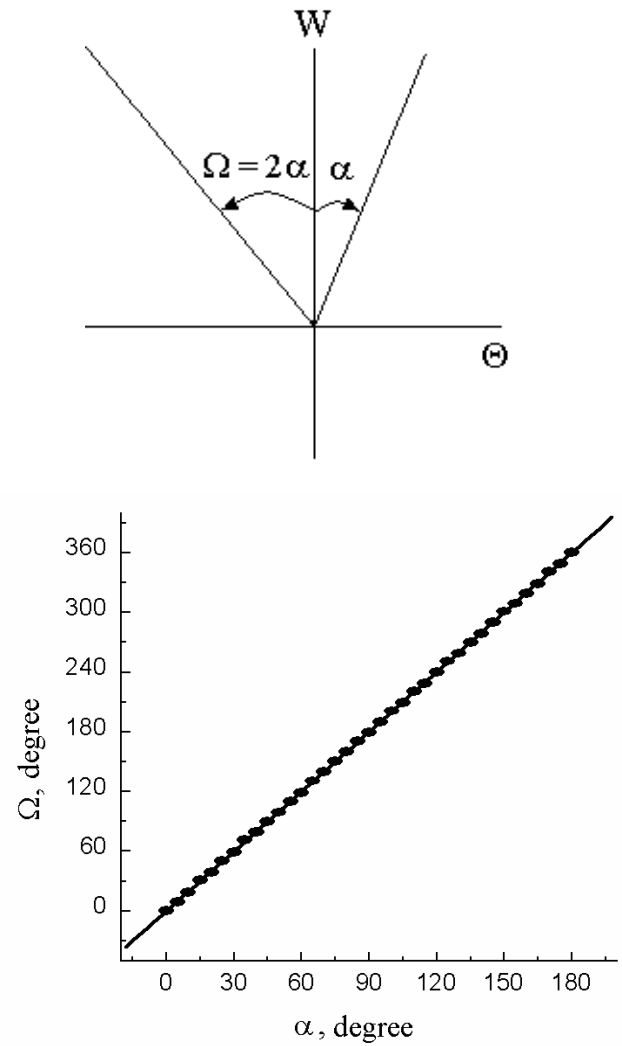

Fig. 6. Influence of the angle of turn for the double Fresnel rhomb on direction (a) and angular position of light polarization plane at the output from the double rhomb (b). $W$ is the datum to count the angle of turn for the double rhomb $(\alpha)$ and polarization plane $(\Omega)$. Points indicate experimental data, the continuous line is an approximation by the formula $\Omega=2 \alpha$. The wavelength $-632.8 \mathrm{~nm}$.

3) Analyzer is inserted. Rotating the analyzer, we find the position when $I$ is maximal. The planes of polarization for polarizer and analyzer are parallel in this case. Fix this angular position of analyzer.

4) Between polarizer and analyzer the double Fresnel rhomb is inserted. Rotating the double Fresnel rhomb, we find the position when $I$ is maximal. In this case, the planes of light polarization at the output of analyzer, double Fresnel rhomb and analyzer are parallel. The determined in this way value $W$ (Fig. 6a) of angular position for the double rhomb is accepted as the initial one.

5) Rotating the double rhomb by some fixed angle $\alpha$ and rotating the analyzer, we find the value of the angle $\Omega$, after reaching which the signal of detector that fixes $I$ is maximal. This angle $\Omega$ shows the angular position of polarization plane on the output of the double Fresnel rhomb. Repeating this procedure for the number of fixed angles $\alpha$, we obtain a tabular function that describes angular dependence of light polarization plane at the output of the double Fresnel rhomb versus the rotation angle for the double Fresnel rhomb (Fig. 6b, points). It is found that at the rotation of the double
Fresnel rhomb by an angle $\alpha$ the plane of light polarization at the output from this double rhomb rotates in the opposite direction by the angle $\Omega=2 \alpha$ (Fig. 6a). To find the angular position of polarization plane between points in this plot, approximation of this dependence by a straight line is carried out. It allows with a least-squares error less than $\pm 0.5^{\circ}$ to assign the position of polarization plane at the output of the double Fresnel rhomb. The testing stages for calibration of the single Fresnel rhomb are as follows: the testing stages of calibration from the first to fifth points are analogous to those when calibrating the double Fresnel rhomb.

6) The single Fresnel rhomb is inserted between the double Fresnel rhomb and analyzer. Adjust the Fresnel rhomb and find the maximal value $I$. In this case, the light beam that goes out of the single rhomb is linearly polarized and its polarization plane is parallel to the plane of polarization for the analyzer.

7) Turn the double Fresnel rhomb by some fixed angle $\alpha$ and find the values of $I$ and $I_{0}$. Repeating this procedure, get the dependence of light transmission for this system versus the rotation angle of the double rhomb (Fig. 7a, points in curve 1). To approximate this dependence, let us use the generalized Malus law [15]
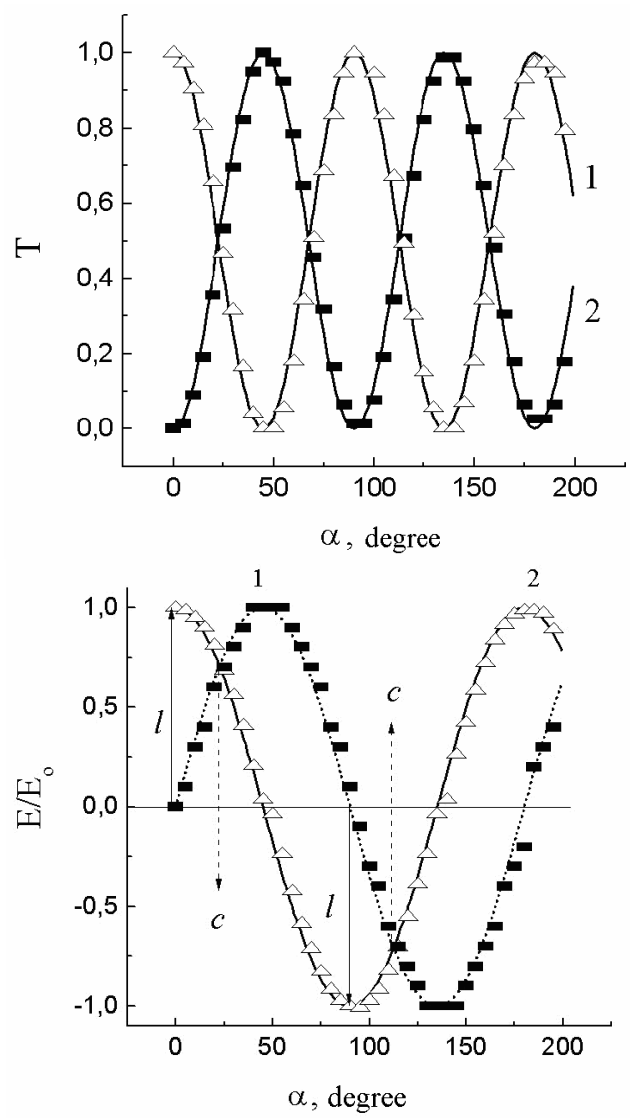

Fig. 7. Dependence of light transmission (a) and electric field (b) on the angle of turn $(\alpha)$ for the double Fresnel rhomb in the system: polarizer, double Fresnel rhomb and analyzer for the parallel (1) and perpendicular (2) orientation of polarization planes for polarizer and analyzer. Light polarization: $l$ - linear, $c$ - circular. 


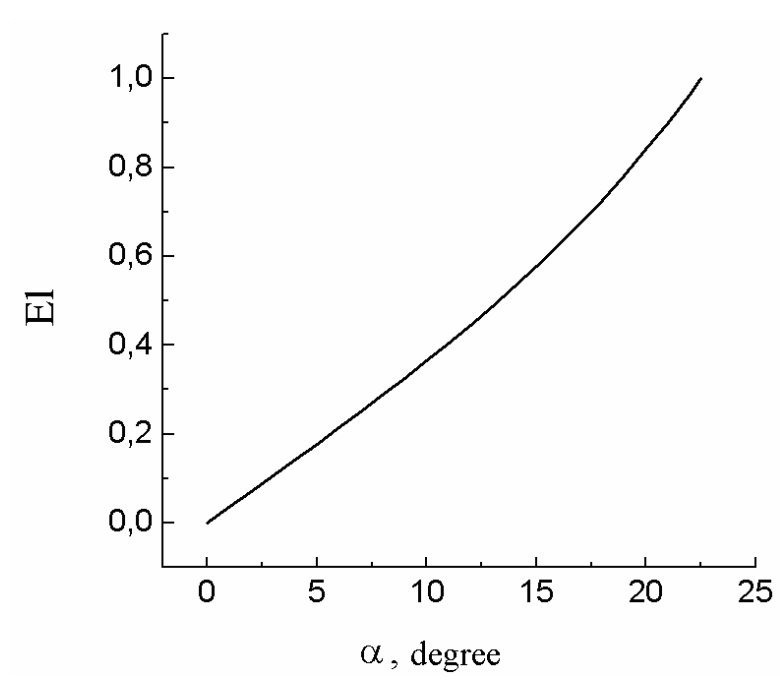

Fig. 8. Ellipticity of light that goes out from the single rhomb versus the rotation angle of the double Fresnel rhomb.

$\frac{T(\alpha)}{T_{0}}=T_{\min }+\left(1-T_{\min }\right) \cos ^{2}(2 \alpha)$,

where $T_{0}$ and $T_{\min }$ are maximum and minimum values of the light transmission.

8) Turn analyzer by $90^{\circ}$ and measure the dependence $T=I / I_{0}$ (Fig. 7a, points in curve 2) versus the rotation angle $\alpha$ of the double Fresnel rhomb and carry out approximation of it using the formula

$$
\frac{T(\theta)}{T_{0}}=T_{\min }+\left(1-T_{\min }\right) \sin ^{2}(2 \alpha) .
$$

The electric field $E$ of an electromagnetic wave is square root of its intensity $(E= \pm \sqrt{A I}$, where $A$ is a constant). Taking advantage of the known values of $I$ and $I_{0}$ and extract the root square of $I$, find the dependence of $E$ on $\alpha$ (Fig. 7b). The dependence of the electric field $E$ at the initial position of analyzer (planes of polarization for the polarizer, double Fresnel rhomb and analyzer are parallel) describes the character of changes of the large axis of polarization ellipse on the rotation angle for the double Fresnel rhomb. The dependence of the electric field $E$ at $90^{\circ}$ position of the analyzer (polarization plane of the analyzer is perpendicular to the planes of polarization for the polarizer and double Fresnel rhomb) describes the character of changes in minor axis of polarization ellipse on the rotation angle for the double Fresnel rhomb. The electric field ratio (more exactly, that for minor-to-large axes of the polarization ellipse) gives the value of the polarization light ellipticity (Fig. 8). At the rotation of the double Fresnel rhomb from $\alpha=0^{\circ}$ to $\alpha=22.5^{\circ}$, the ellipticity changes from 0 to 1 . When $\alpha=0^{\circ}$, we have plane-polarized light and at $\alpha=22.5^{\circ}$ - circularly polarized light (Fig. 7) with clockwise rotation. At further rotation of the double Fresnel rhomb, linear polarization is realized at $\alpha=90^{\circ}$, and circular polarization at $\alpha=112.5^{\circ}$ with anticlockwise rotation.

\section{Conclusions}

Performed in this work is the analysis of properties inherent to wave plates and Fresnel rhombi. It has been shown that the Fresnel rhomb should be preferably used to control light polarization of powerful laser sources that generate light in a wide spectral range. The technical parameters of the rhomb have been estimated. The calibration method for the single and double rhombi is analyzed. It should be noted that the Fresnel rhomb possesses high stability against the temperature, in respect of surrounding environment, errors of adjustment, variations of the wavelength; therefore, the Fresnel rhomb is used in polarimeters [13], including the facility intended for researches of magnetic fields and atmosphere of stars [11].

\section{References}

1. P.J. Maguire, L.P. Barry, T. Krug, W.H. Guo, J. O’Dowd, M. Lynch, A.L. Bradley, J.F. Donegan // J. Lightwave Technology 24(7), p. 2683 (2006).

2. X. Zhang, M. Izutsu // Technical Report of IEICE. OPE 98, No. 166 (19980703), p. 19-24.

3. T.K. Liang, L.R. Nunes, M. Tsuchiya, K.S. Abedin, T. Miyazaki, D. Van Thourhout, W. Bogaerts, P. Dumon, R. Baets, and H.K. Tsang // Opt. Communs 265(1), p. 171 (2006).

4. J. Montoya, Q. Hu // J. Appl. Phys. 95(5), p. 2230 (2004).

5. F. Cannone, G. Chirico, G. Baldini, A. Dlalaspro // J. Microscopy 210(2), p. 149 (2003).

6. C.H. Lee, S. Jayaraman // Optical and Quantum Electronics 6(1), p. 115 (1974).

7. OOO "ELAN+", Russia, 190103, St-Petersburg, per. Derptskiy, 3. (in Russian).

8. CASIX Inc., P.O. Box 1103, Fuzhou, Fujian 350014, China.

9. B. Halle Nachfl. GmbH, Hubertusstraße 10, D12163, Berlin, Germany.

10. DORIC LENSES INC 357 rue Franquet Quebec, QC, G1P 4N7, Canada.

11. P. Petit, J.-F. Donati and the ESPaDOnS project team, Stellar polametry with ESPaDOnS // arXiv:astro-ph/0403118v1 4 Mar 2004, p. 1-8.

12. Meadowlark Optics, Inc., 5964 Iris Parkway, PO Box 1000, Frederick, CO, 80530, USA.

13. P.A. Williams, A.H. Rose, C.M. Wang // Appl. Opt. 36(25), p. 6466 (1997).

14. D. Mawet, C. Hanot, C. Lenaerts, P. Riaud, D. Defrere, D. Vandormael, J. Loicq, K. Fleury, J.-Y. Plesseria, J. Surdej, S. Habraken // Opt. Express 15(20), p. 12850 (2007).

15. R. Ditchbern, Physical Optics. Nauka, Moscow, 1965. 631 p. (in Russian). 
16. EKSPLA UAB, Savanoriu Av. 231, LT-02300, Vilnius-53, Lithuania.

17. K.I. Tarasov, Spectral Devices. Mashinostroeniye Publ., Leningrad, 1977. (in Russian).

18. Newlight Photonics, P.O. Box 261, Toronto Postal STN "E", 772 Dovercourt Road, Toronto, ON 6H 4E2, Canada.

19. 20. Halbo Optics Fresnel Rhombs.

20. ZAO "Opto-Tekhnologicheskaya Laboratoriya" 195197, Russia, St-Peterburg, Polustrovsky pr., 61.
21. UQG Ltd The Norman Industrial Estate, 99-101 Cambridge Road, Milton, Cambridge, CB4 6AT, England.

22. OOO Elektrosteklo. 119571 Moscow, prospect Vernadskogo, 113-106 (Russia).

23. II-VI INFRARED 375, Saxonburg Blvd, Saxonburg, PA 16056. 